\title{
Simplifying the assessment of coronary artery stenosis by enhancing instantaneous wave free ratio
}

\author{
Vincent Spagnoli ${ }^{1}$, Fabien Picard ${ }^{1}$, Victor-Xavier Tadros ${ }^{1}$, Daniel Cournoyer ${ }^{2}$, Serge Doucet ${ }^{1}$, Jean \\ François Tanguay ${ }^{1}$, Gilbert Gosselin ${ }^{1}$, Pierre de Guise ${ }^{1}$, Richard Gallo ${ }^{1}$ \\ ${ }^{1}$ Department of Cardiology, Montréal Heart Institute, Université de Montréal, Montréal, Canada; ${ }^{2}$ Montréal Heart Institute Coordinating Center, \\ Montréal, Canada \\ Contributions: (I) Conception and design: V Spagnoli, R Gallo; (II) Administrative support: V Spagnoli, R Gallo; (III) Provision of study materials or \\ patients: V Spagnoli, R Gallo; (IV) Collection and assembly of data: V Spagnoli, R Gallo; (V) Data analysis and interpretation: V Spagnoli, R Gallo, \\ D Cournoyer; (VI) Manuscript writing: All authors; (VII) Final approval of manuscript: All authors. \\ Correspondence to: Richard Gallo, MD. Department of Cardiology, Montréal Heart Institute, Université de Montréal, 5000 Belanger Street, Montréal, \\ Quebec, Canada. Email: gallor@me.com.
}

Background: Instantaneous wave free ratio (iFR) does not require adenosine, but has a relatively wide intermediate range where functional assessment remains inconclusive. In this pilot study, we sought to enhance iFR through with the use of intracoronary (IC) saline (iFRs) and contrast media (iFRc) and determine whether these techniques correlated well with fractional flow reserve (FFR).

Methods: Patients with coronary artery stenosis (CAS) associated with an iFR in the intermediate zone $(\geq 0.86$ and $\leq 0.93)$ were prospectively assessed with resting distal coronary pressure/aorta pressure $(\mathrm{Pd} / \mathrm{Pa})$, iFR, iFRs, iFRc and FFR.

Results: A total of 40 coronary lesions were studied (40 patients). Pearson correlation coefficients for FFR and iFR, FFR and iFRs, FFR and iFRc were respectively: $0.57(\mathrm{P}=0.0002), 0.80(\mathrm{P}<0.0001)$ and 0.77 $(\mathrm{P}<0.0001)$. Receiver-operating characteristic $(\mathrm{ROC})$ curve analysis showed similar area under the curve (AUC) of iFRs and iFR [0.90 (95\% CI: 0.76-1) vs. 0.89 (95\% CI: 0.79-0.99), P=0.89]. Youden's index established cut-off values of $\leq 0.90$ for iFR (sensitivity $=91 \%$, specificity $=74 \%$ ) and $\leq 0.78$ for iFRs (sensitivity $=73 \%$, specificity $=100 \%$ ). In contrast, the AUC of iFRc was superior to the AUC of iFR [0.99 (95\% CI: 0.98-1), P=0.049]. iFRc showed excellent accuracy and established cut-off values of $\leq 0.81$ in predicting an FFR value of $\leq 0.80$ (sensitivity $=100 \%$, specificity $=93 \%$ ).

Conclusions: When iFR is in the intermediate zone, functional assessment of CAS by iFR is enhanced with the use of contrast media but not saline. This pilot study could be hypothesis generating for further study to enhance iFR specificity and sensibility.

Keywords: Instantaneous wave free ratio (iFR); fractional flow reserve (FFR); contrast media; saline solution; coronary artery disease

Submitted Oct 19, 2017. Accepted for publication Dec 11, 2017.

doi: $10.21037 / c d t .2017 .12 .04$

View this article at: http://dx.doi.org/10.21037/cdt.2017.12.04

\section{Introduction}

Determining the physiologic significance of coronary artery stenosis (CAS) by coronary angiography has significant limitations (1). Fractional flow reserve (FFR) has emerged as an invasive tool to help identify hemodynamically significant coronary lesions and is commonly used in clinical practice. Its clinical relevance as a predictor of major outcomes has been clearly demonstrated (2-4).

Instantaneous wave free ratio (iFR) is a newer method introduced to measure the functional impact of an intermediate CAS under resting conditions without the need to induce a hyperaemic reaction $(5,6)$. iFR isolates 
a specific period in diastole, called the wave-free period, and uses the ratio of distal coronary pressure $(\mathrm{Pd})$ to the pressure observed in the aorta $(\mathrm{Pa})$ over this period. During this period, pressure and flow are linearly related (wave free) as compared to the rest of the cardiac cycle resulting in a constant coronary resistance (5). Large and retrospective comparisons of iFR $v s$. the more traditional FFR initially showed conflicting results regarding the correlation between iFR and FFR. Whereas the ADenosine Vasodilator Independent Stenosis Evaluation (ADVISE) registry demonstrated an excellent correlation between iFR and FFR (7), the Verification of Instantaneous Wave-Free Ratio and Fractional Flow Reserve for the Assessment of Coronary Artery Stenosis Severity in Everyday Practice (VERIFY) study failed to show such a correlation (8). In order to overcome limitations and methodological heterogeneity of prior comparative studies, the ADVISE II trial was designed to assess the diagnostic accuracy of iFR in a prospective, independent core laboratory-based environment (9). This study showed a strong correlation between iFR and FFR when iFR values were $<0.86$ or $>0.93$. However, there was a poor correlation when lesions fell within an intermediate range of $\mathrm{iFR} \geq 0.86$ and $\leq 0.93$. When lesions fell within this intermediate range decisions regarding physiological significance of the lesion could not be determined. This situation arises in approximately $30 \%$ of cases necessitating that the operator reverts to an adenosine mediated FFR assessment. Therefore, relying on these good correlations with FFR, this hybrid strategy was recommended.

Adenosine is the gold standard to induce hyperaemia in FFR procedures (10). However, its use is associated with hemodynamic and arrhythmic side effects, entails additional costs and becomes time consuming. Saline solution and contrast media have also been shown to increase shear stress and ischemia induced vasodilatation $(11,12)$. They may represent in many situations a simple, safe, less costly, readily available and instantaneously accessible alternative to adenosine in functional assessment of CAS.

In this study, we sought to determine whether iFR with an additional saline injection (iFRs) or iFR with an additional contrast injection (iFRc) can be used as an alternative to adenosine mediated FFR when the functional assessment of a coronary stenosis falls within the intermediate (grey zone) of standard iFR. We also sought to establish a threshold value for functional significance of a coronary lesion using iFRs and iFRc in comparison to adenosine mediated FFR.

\section{Methods}

\section{Study population}

This study was prospectively conducted between September 2015 and September 2016 at Montréal Heart Institute, Montréal, Canada. Patients referred for coronary angiography were deemed eligible if the presence of a CAS warranted an iFR or FFR measurement. Only patients with an intermediate (inconclusive) $\mathrm{iFR} \geq 0.86$ and $\leq 0.93$ were included in this study. Exclusion criteria included the following: ST-segment elevation myocardial infarction, functional test on a culprit lesion in non-ST-segment elevation myocardial infarction patients, hemodynamically unstable patients, uncompensated heart failure and risk of contrast induced nephropathy (creatinine clearance $\leq 60 \mathrm{~mL} / \mathrm{min}$ ). The study was approved by our local ethics review board (registration number 2015-1837). Written informed consent was obtained from all patients.

\section{iFR and FFR procedures}

Coronary artery lesions were evaluated using a 0.014 -inch pressure wire (ComboWire XT, Volcano Corporation, San Diego, California, USA), introduced through a 6-F guiding catheter into the coronary artery. The guide wire was advanced at least $2 \mathrm{~cm}$ distally to the target lesion. Distal coronary and aortic pressures were recorded at baseline and during induction of hyperemia. Continuous pressure signals and electrocardiogram monitoring was performed and digitally stored. Intra-coronary nitroglycerin $1 \mathrm{mg}$ was given at the beginning of every procedure. Heart rate and arterial pressure were recorded at the moment of each iFR, iFRs, iFRc and FFR evaluation.

Measurements were recorded as follows: (I) resting $\mathrm{Pd} / \mathrm{Pa}$; (II) standard iFR; (III) iFRs after an intracoronary (IC) injection of saline solution $(10 \mathrm{~mL})$; (IV) iFRc after an IC injection of nonionic iso-osmolar contrast agent, Iodixanol $\left(\right.$ Visipaque $\left.^{\circledR}\right)(8 \mathrm{~mL})$; (V) FFR after $240 \mu \mathrm{g}$ IC injection of adenosine.

Measurements of iFRs or iFRc were started one heart beat following bolus administration. FFR was started three heart beats following bolus administration. A continuous measurement was made with the guiding catheter disengaged from the coronary ostium and stopped when the pressure curve returned to the baseline value. Duplicate measurements were performed in order to evaluate reproducibility. An FFR value $\leq 0.8$ was used to define a significant CAS with hemodynamic impairment (3). 
Table 1 Baseline demographic and clinical characteristics of patients

\begin{tabular}{|c|c|}
\hline Clinical characteristics & Frequency [n (\%)] \\
\hline Number of patients & 40 \\
\hline \multicolumn{2}{|l|}{ Demographic characteristics } \\
\hline Age (years) (mean \pm SD) & $66 \pm 8$ \\
\hline Male & 35 [88] \\
\hline \multicolumn{2}{|l|}{ Clinical characteristics } \\
\hline Smoking & $10[25]$ \\
\hline Diabetes mellitus & 17 [43] \\
\hline Hypertension & 33 [83] \\
\hline Dyslipidemia & 30 [75] \\
\hline Previous revascularization & 15 [38] \\
\hline \multicolumn{2}{|l|}{ Medication } \\
\hline Aspirin & 39 [98] \\
\hline Clopidogrel & 22 [55] \\
\hline Beta-blockers & $27[68]$ \\
\hline RAA antagonists & 22 [55] \\
\hline Calcium-channel blockers & $8[20]$ \\
\hline Statins & 37 [93] \\
\hline LVEF (\%) (mean \pm SD) & $57 \pm 8$ \\
\hline \multicolumn{2}{|l|}{ Indication for angiography } \\
\hline Stable angina/silent ischemia & 29 [73] \\
\hline Unstable angina & $11[28]$ \\
\hline Creatinin $(\mu \mathrm{mol} / \mathrm{L})($ mean $\pm \mathrm{SD})$ & $85 \pm 24$ \\
\hline
\end{tabular}

$\mathrm{SD}$, standard deviation; LVEF, left ventricular ejection fraction; RAA, renin-angiotensin-aldosterone.

\section{Angiographic characteristics and stenosis evaluation}

Data on the number of diseased vessels, target vessels, lesion locations and percent diameter stenosis were collected. Subsequent procedural decisions were left to the operator's discretion.

\section{Statistical analysis}

The data are expressed as mean \pm standard deviation (SD). The reproducibility of iFRs and iFRc was assessed, using the intra-class correlation coefficient with $95 \%$ CI. Univariate correlations between continuous variables were assessed by Pearson's correlation (r). Receiver-operating
Table 2 Angiographic and procedural characteristics

\begin{tabular}{lc}
\hline Angiographic characteristics & Frequency \\
\hline Number of lesions & 40 \\
Number of diseased vessels & $20[50]$ \\
1 & $11[28]$ \\
2 & $9[22]$ \\
3 & \\
Target vessel FFR & $34[85]$ \\
LAD & $4[10]$ \\
Circumflex & $2[5]$ \\
RCA & \\
Lesion location & $11[28]$ \\
Proximal & $26[65]$ \\
Mid & $3[7]$ \\
Distal & $49 \pm 11$ \\
Contrast volume & $180 \pm 64$ \\
Rate of stenting & $21[53]$ \\
\hline Values are expressed visual estimation & \\
\hline
\end{tabular}

Values are expressed as mean \pm SD or $n$ [\%]. FFR, fractional flow reserve; $L A D$, left anterior descending; $R C A$, right coronary artery; IQR, interquartile range.

characteristic (ROC) curves analysis was performed to estimate the diagnostic performance of iFR, iFRs and iFRc. Comparison of ROC curves analysis were made and a $\mathrm{P}$ value $<0.05$ was considered significant. Youden's index was used to determine the optimal threshold value of iFR, iFRs and iFRc. Sensitivity, specificity, negative predictive value (NPV), positive predictive value (PPV) of iFR, iFRs and iFRc using a FFR value $\leq 0.8$ as a reference value were calculated and $95 \%$ CI were reported. The data analysis was generated using SAS/STAT software, version 9.4.

\section{Results}

A total of 40 coronary lesions in 40 patients were included in the present study. Baseline clinical, lesion and procedural characteristics are reported in Tables 1,2.

\section{Reproducibility and correlation}

\section{Reproducibility}

Reproducibility of iFRs and iFRc showed an excellent intra- 


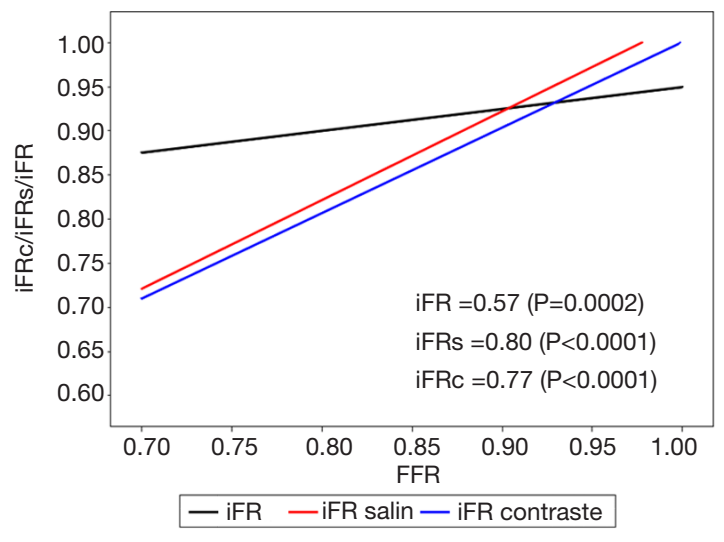

Figure 1 Pearson's correlation $r$ analysis between iFR and FFR, iFRs and FFR, iFRc and FFR. iFR, instantaneous wave free ratio; iFRc, iFR with additional contrast injection; iFRs, iFR with additional saline injection; FFR, fractional flow reserve.

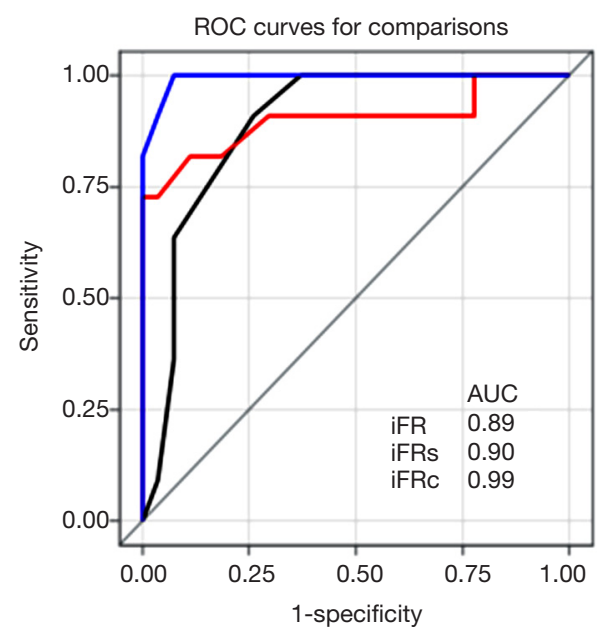

Figure 2 ROC curve analysis of iFR, iFRs and iFRc. ROC, receiver-operating characteristic; AUC, area under the curve; iFR, instantaneous wave free ratio; iFRc, iFR with additional contrast injection; iFRs, iFR with additional saline injection.

class correlation coefficient of $0.94(0.88-0.97)$ and 0.92 (0.85-0.96) respectively.

\section{Correlation between iFR, iFRs and iFRc with FFR}

As expected, an inconclusive iFR (intermediate range value), showed a poor correlation with FFR values: $r=0.57$ $(\mathrm{P}=0.0002)$. In contrast, iFRs and iFRc showed a strong correlation with FFR: $r=0.80(\mathrm{P}<0.0001)$ and $r=0.77$ $(\mathrm{P}<0.0001)$ respectively (Figure 1$)$.

\section{ROC curves analysis and threshold values}

ROC curve analysis demonstrated an area under the curve (AUC) for iFR at 0.89 (95\% CI: 0.79-0.99) (Figure 2). Youden's index established an iFR cut-off at 0.90 , sensitivity $=91 \%(95 \%$ CI: $59-100 \%)$, specificity $=74 \%$ (95\% CI: 54-89\%). Using this threshold value, lesions were incorrectly classified in $23 \%$ of the cases, confirming the necessity of additional adenosine mediated FFR testing for intermediate iFR lesions.

ROC curve analysis of iFRs showed a good accuracy for predicting FFR values $\leq 0.80$ with an AUC of 0.90 (95\% CI: 0.76-1) (Figure 2). There was no statistical difference between the ROC curve of iFR and the ROC curve of iFRs: 0.89 vs. $0.90, \mathrm{P}=0.89$. Youden's index testing established a threshold value of $\leq 0.78$ for iFRs, sensitivity $=73 \%$ ( $95 \%$ CI: $39-94 \%)$, specificity $=100 \%$ (95\% CI: $87-100 \%)$. This threshold value correctly classified $92 \%$ of subjects.

ROC curve analysis of iFRc demonstrated an excellent accuracy with an AUC of 0.99 (95\% CI: 0.98-1) (Figure 2). There was a statistical difference between the ROC curve of iFRc and the ROC curve of iFR: 0.99 vs. $0.89, \mathrm{P}=0.049$. Youden's index established a threshold value of $\leq 0.81$ for iFRc, sensitivity $=100 \%$ (95\% CI: $72-100 \%)$, specificity $=93 \%$ (95\% CI: 76-99\%). Compared to adenosine mediated FFR, $95 \%$ of subjects were positively identifying (Figure 3). Sensitivity, specificity, PPV and NPV of each method are summarized in Table 3.

\section{Discussion}

In this study, we sought to determine whether the sensitivity and specificity of an intermediate (inconclusive) iFR measurement could be enhanced by iFRs or iFRc and whether these simple procedures can be used as an alternative to adenosine mediated FFR. The main findings of our study can be summarized as follows: (I) when iFR is in the intermediate zone, iFRc is predictive of an adenosine FFR value under 0.80 and potentially represents a simple alternative to FFR; (II) iFRc is reproducible and showed an excellent correlation with adenosine FFR when iFR is in the intermediate zone; (III) an iFRc threshold value of $\leq 0.81$ can predict a positive FFR value $(\leq 0.80)$; (IV) in contrast, iFR accompanied by IC injection of saline did not demonstrate a statistically significant correlation compared to adenosine mediated FFR.

The functional and invasive assessment of CAS is based on a constant and minimized myocardial resistance. 


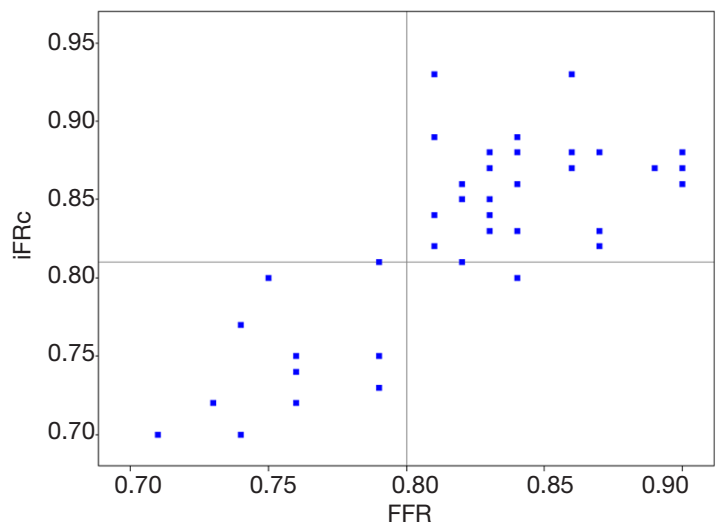

Figure 3 Correlation between iFRc and FFR with respective threshold values. iFRc, iFR with additional contrast injection; FFR, fractional flow reserve.

Currently, two methods are used in modern catheterization laboratories: FFR and iFR. The former is the established gold standard and is calculated during hyperaemia, originally obtained with adenosine, and averaged through several cardiac cycles. In order to facilitate FFR adoption, two different approaches have been proposed: intra coronary adenosine administration (13), and more recently introduction of contrast medium as an alternative hyperemic agent. Contrast medium induced FFR, studies have shown a good correlation between contrast induced $\mathrm{Pd} / \mathrm{Pa}$ and adenosine mediated FFR (14-17).

The cornerstone of the iFR is based on the identification of a period in diastole with a natural, constant and minimized resistance represented by an almost quiescent wave free period (8). Introduced by Sen and colleagues in 2012, this method avoids the use of pharmacologic agents such as adenosine to induce hyperaemia. Consequently, iFR represents an interesting alternative to FFR for functional assessment of CAS (5).

The diagnostic value of iFR in identifying CAS with an FFR $\leq 0.80$ varied between studies. In the ADVISE registry, which looked at 339 coronary stenosis, the cutoff value for iFR was 0.89 and accuracy was calculated at $94 \%$ (7). In the prospective VERIFY study which included 206 prospectively studied patients and 500 retrospective analysis of pressure recordings, the diagnostic accuracy of an iFR value of $\leq 0.80$, was only $60 \%$ when compared to the FFR cut-off value of $\leq 0.80$ (8). Similarly, a retrospective analysis from the RESOLVE study analyzing 1,593 coronary lesions established an iFR cut-off of 0.90 with a diagnostic accuracy of only $81.5 \%$ (18). Wide methodological differences may explain the conflicting results between threshold values and accuracy. In order to overcome this limitation, Escaned et al. demonstrated that a hybrid iFR-FFR decision-making process can be used to improve diagnostic accuracy (9). They found a very good correlation when iFR was $<0.86$ or $>0.93$ with a PPV $=93 \%$ and a NPV $=95 \%$ compared to adenosine mediated FFR. A poor correlation was found when lesions fells within the iFR intermediate zone $(\geq 0.86$ and $\leq 0.93)$. This situation occurred in $30 \%$ of cases, requiring additional adenosine mediated FFR testing.

Adenosine during iFR did not improve the diagnostic accuracy when compared to iFR in a study involving 51 patients (19). Unlike our study where we focused only on intermediate lesions, all lesion types were considered in the former study. This may have contributed to the absence of observed diagnostic accuracy.

A major shortcoming of iFR, is its large intermediate or inconclusive zone as compared to FFR. The adoption of iFR in clinical practice could be significantly improved, by reducing the need of reverting to adenosine mediated FFR. We hypothesized that an alternative, less expensive, readily available and safe pharmacologic hyperaemic agents could improve the diagnostic accuracy of iFR by enhancing the quiescent diastolic wave free period. Saline solution and contrast media represent such agents. Saline solution (isotonic or hypertonic) has never been evaluated in the functional assessment of CAS despite his wellknown effect on coronary artery blood flow. Saline solution decreases coronary resistances and increases vasodilatation $(11,12)$. Contrast media, on the other hand has already been evaluated as a hyperemic agent in FFR (14-17). In one multicenter study, adjudicated by a central core laboratory, contrast mediated FFR compared favorably to adenosine mediated FFR and was superior to iFR for predicting CAS severity (15). This study differs from our study in that all coronary lesions were included for analysis regardless if values were in the intermediate (inconclusive) zone. Recently, contrast media induced $\mathrm{Pd} / \mathrm{Pa}$ during end-diastolic FFR was shown to offer a better diagnostic performance than contrast induced FFR (20). However, this calculation is a tedious and a labor-intensive process that limits its acceptance in everyday clinical practice. Since iFR is focused on a very narrow period of the diastolic phase (beginning $25 \%$ of the way into diastole and ending $5 \mathrm{~ms}$ before the end of diastole), hyperaemia induced iFR may provide a standardized easily reproducible alternative.

However, despite the large intermediate and inconclusive zone of iFR as compared to FFR, clinical outcome associated 
Table 3 Sensitivity, specificity, PPV and NPV of iFR, iFRs and iFRc when compared to FFR values (significant lesion $\leq 0.80$ ) (95\% CI)

\begin{tabular}{lccc}
\hline iFR accuracy & iFR $\leq 0.90[\%]$ & iFRs $\leq 0.78[\%]$ & iFRc $\leq 0.81[\%]$ \\
\hline Sensitivity & $91[59-100]$ & $73[39-94]$ & $100[72-100]$ \\
Specificity & $74[54-89]$ & $100[87-100]$ & $93[76-99]$ \\
PPV & $59[33-82]$ & $100[63-100]$ & $85[55-98]$ \\
NPV & $95[76-100]$ & $90[73-98]$ & $100[86-100]$ \\
\hline
\end{tabular}

iFR, instantaneous wave free ratio; iFRc, iFR with additional contrast injection; iFRs, iFR with additional saline injection; PPV, positive predictive value; NPV, negative predictive value; FFR, fractional flow reserve.

with the use of iFR have recently been investigated in two multicenter, randomized and controlled trials $(21,22)$. In patients with stable or acute coronary syndrome, these studies showed that an iFR-guided revascularization (using a threshold value of 0.89 ) was non-inferior to FFRguided revascularization. The rate of major adverse cardiac events at 12 months were similar between the two groups. Nevertheless, FFR is derived from comparison with several non-invasive evaluations of ischemic heart disease and the clinical outcome and longer follow-up have been evaluated in several trials. Whether interventional cardiologists will rely solely on iFR in regard of these two studies, using a single threshold for iFR despite the poor correlation with FFR when in the grey zone remain unknown. Our study could also be hypothesis generating for further studies to evaluate whether iFRc could enhance iFR specificity and sensibility when using a single threshold of 0.89 .

Alternatively of invasive functional assessment of CAS, a number of approaches have been proposed to derive a virtual FFR that would be computed from various image acquisition techniques including cardiac computed tomography (23) or diagnostic coronary angiography, in combination with computational algorithms, rather than measured with a pressure wire. While acceptable correlation has been reported, three-dimensional quantitative coronary angiography provided a higher image resolution and appeared to be superior to coronary computed tomography angiography for functional evaluation (24). Indeed, two ongoing trials (FAVOR Europe Japan and FAVOR China) also based on quantitative flow ratio (QFR), will evaluate the diagnostic accuracy and determine the presence or absence of hemodynamically-significant CAS of QRF when compared to FFR as the reference standard. In the future, this technique could avoid the need for pressure wire evaluation. Nevertheless, further studies are required to determine the clinical and economic benefit of such imaging evaluation.

\section{Limitations}

Although prospective and methodologically rigorous, this remains a single center study with a small cohort of patients. This small cohort could explain why iFRs correlated well with FFR but did not have significantly improved diagnostic accuracy as compared to iFRc. Also, despite meticulous reporting and collection of data, a blinded core laboratory did not independently analyze the data. A single $10 \mathrm{~mL}$ dose of saline solution and $8 \mathrm{~mL}$ of contrast media were used and dose-effect analysis with different amounts on iFRs or iFRc was not performed. This choice was supported by the simplicity of use and based on previous studies involving contrast induced $\mathrm{Pd} / \mathrm{Pa}$ where volumes of 6 to $10 \mathrm{~mL}$ of contrast was used. In addition, there was no correlation with non-invasive stress-testing or imaging to document the ability to identify ischemia-generating stenosis nor was there any clinical correlation to these physiological findings. Since this was planned as a pilot study to improve on an iFR technique, we did not collect data on patients who had positive or negative iFR values. Therefore, the proportion of patients that fell into the intermediate zone is not reported. As a result of the relatively low number of lesions studied, we cannot account for the heterogeneity in lesion location or lesion related factors such as bifurcation lesions, calcifications, level of disease, etc.

\section{Conclusions}

Functional assessment of coronary artery lesions by iFR is enhanced by the use of IC contrast media and compares favorably to adenosine mediated FFR. In patients where functional assessment by iFR falls within an intermediate value, injection of contrast media followed by an immediate iFR can predict lesion severity as well as adenosine mediated FFR. Our study could be hypothesis generating for further studies to evaluate whether iFRc could enhance 
iFR specificity and sensibility when using a single threshold of 0.89 .

\section{Acknowledgements}

V Spagnoli, F Picard and VX Tadros have recieved research grants from French Federation of Cardiology.

\section{Footnote}

Conflicts of Interest: The authors have no conflicts of interest to declare.

Ethical Statement: The study was approved by the local institutional review board (No. 2015-1837) and written informed consent was obtained from all patients.

\section{References}

1. White CW, Wright CB, Doty DB, et al. Does visual interpretation of the coronary arteriogram predict the physiologic importance of a coronary stenosis? $\mathrm{N} \mathrm{Engl} \mathrm{J}$ Med 1984;310:819-24.

2. Pijls NH, van Schaardenburgh P, Manoharan G, et al. Percutaneous coronary intervention of functionally nonsignificant stenosis: 5-year follow-up of the DEFER Study. J Am Coll Cardiol 2007;49:2105-11.

3. Tonino PA, De Bruyne B, Pijls NH, et al. Fractional flow reserve versus angiography for guiding percutaneous coronary intervention. N Engl J Med 2009;360:213-24.

4. De Bruyne B, Pijls NH, Kalesan B, et al. Fractional flow reserve-guided PCI versus medical therapy in stable coronary disease. N Engl J Med 2012;367:991-1001.

5. Sen S, Escaned J, Malik IS, et al. Development and validation of a new adenosine-independent index of stenosis severity from coronary wave-intensity analysis: results of the ADVISE (ADenosine Vasodilator Independent Stenosis Evaluation) study. J Am Coll Cardiol 2012;59:1392-402.

6. Picard F, Pighi M, Ly HQ. Fractional flow reserve and resting indices for coronary physiologic assessment: Practical guide, tips, and tricks. Catheter Cardiovasc Interv 2017;90:598-611.

7. Petraco R, Escaned J, Sen S, et al. Classification performance of instantaneous wave-free ratio (iFR) and fractional flow reserve in a clinical population of intermediate coronary stenoses: results of the ADVISE registry. EuroIntervention 2013;9:91-101.
8. Berry C, van 't Veer M, Witt N, et al. VERIFY (VERification of Instantaneous Wave-Free Ratio and Fractional Flow Reserve for the Assessment of Coronary Artery Stenosis Severity in EverydaY Practice): a multicenter study in consecutive patients. J Am Coll Cardiol 2013;61:1421-7.

9. Escaned J, Echavarría-Pinto M, Garcia-Garcia HM, et al. Prospective Assessment of the Diagnostic Accuracy of Instantaneous Wave-Free Ratio to Assess Coronary Stenosis Relevance: Results of ADVISE II International, Multicenter Study (ADenosine Vasodilator Independent Stenosis Evaluation II). JACC Cardiovasc Interv 2015;8:824-33.

10. De Bruyne B, Pijls NH, Barbato E, et al. Intracoronary and intravenous adenosine 5 '-triphosphate, adenosine, papaverine, and contrast medium to assess fractional flow reserve in humans. Circulation 2003;107:1877-83.

11. Crystal GJ, Gurevicius J, Kim SJ, et al. Effects of hypertonic saline solutions in the coronary circulation. Circ Shock 1994;42:27-38.

12. Baile EM, Paré PD, D'yachkova $Y$, et al. Effect of contrast media on coronary vascular resistance: contrast-induced coronary vasodilation. Chest 1999;116:1039-45.

13. Khashaba A, Mortada A, Omran A. Intracoronary versus intravenous adenosine-induced maximal coronary hyperemia for fractional flow reserve measurements. Clin Med Insights Cardiol 2014;8:17-21.

14. Leone AM, Scalone G, De Maria GL, et al. Efficacy of contrast medium induced $\mathrm{Pd} / \mathrm{Pa}$ ratio in predicting functional significance of intermediate coronary artery stenosis assessed by fractional flow reserve: insights from the RINASCI study. EuroIntervention 2015;11:421-7.

15. Johnson NP, Jeremias A, Zimmermann FM, et al. Continuum of vasodilator stress from rest to contrast medium to adenosine hyperemia for fractional flow reserve assessment. JACC Cardiovasc Interv 2016;9:757-67.

16. Spagnoli V, Amabile N, Dillinger JG, et al. Myocardial fractional flow reserve measurement using contrast media as a first-line assessment of coronary lesions in current practice. Can J Cardiol 2016;32:739-46.

17. Leone AM, Martin-Reyes R, Baptista SB, et al. The Multi-center Evaluation of the Accuracy of the Contrast MEdium INduced Pd/Pa RaTiO in Predicting FFR (MEMENTO-FFR) Study. EuroIntervention 2016;12:708-15.

18. Jeremias A, Maehara A, Généreux P, et al. Multicenter core laboratory comparison of the instantaneous wave-free ratio and resting $\mathrm{Pd} / \mathrm{Pa}$ with fractional flow reserve: the 
RESOLVE study. J Am Coll Cardiol 2014;63:1253-61.

19. Sen S, Asrress KN, Nijjer S, et al. Diagnostic classification of the instantaneous wave-free ratio is equivalent to fractional flow reserve and is not improved with adenosine administration. Results of CLARIFY (Classification Accuracy of Pressure-Only Ratios Against Indices Using Flow Study). J Am Coll Cardiol 2013;61:1409-20.

20. Kanaji Y, Murai T, Lee T, et al. Efficacy of pressure parameters obtained during contrast medium-induced submaximal hyperemia in the functional assessment of intermediate coronary stenosis in comparison with instantaneous wave-free ratio. Int J Cardiol 2016;208:128-36.

21. Davies JE, Sen S, Dehbi HM, et al. Use of the Instantaneous Wave-free Ratio or Fractional Flow Reserve in PCI. N Engl J Med 2017;376:1824-34.

22. Götberg M, Christiansen EH, Gudmundsdottir IJ, et al. Instantaneous Wave-free Ratio versus Fractional Flow Reserve to Guide PCI. N Engl J Med 2017;376:1813-23.

23. Danad I, Szymonifka J, Twisk JW, et al. Diagnostic performance of cardiac imaging methods to diagnose ischaemia-causing coronary artery disease when directly compared with fractional flow reserve as a reference standard: a meta-analysis. Eur Heart J 2017;38:991-8.

24. Liu L, Yang W, Nagahara Y, et al. The impact of image resolution on computation of fractional flow reserve: coronary computed tomography angiography versus 3-dimensional quantitative coronary angiography. Int $\mathbf{J}$ Cardiovasc Imaging 2016;32:513-23.
Cite this article as: Spagnoli V, Picard F, Tadros VX, Cournoyer D, Doucet S, Tanguay JF, Gosselin G, de Guise P, Gallo R. Simplifying the assessment of coronary artery stenosis by enhancing instantaneous wave free ratio. Cardiovasc Diagn Ther 2018;8(2):156-163. doi: 10.21037/cdt.2017.12.04 